\title{
Diagnostic approach to interstitial lung disease
}

\author{
Athena Gogali • Athol U. Wells
}

Published online: 15 September 2012

(C) Springer Science+Business Media, LLC 2012

\begin{abstract}
Interstitial lung disease includes a variety of entities with strikingly diverse natural histories and treated outcomes. Thus, in clinical practice, an accurate diagnosis is essential in order to construct an optimal treatment strategy. The gold standard for diagnosis is now a multi-disciplinary diagnosis, with participation by clinicians, radiologists, and, when applicable, histopatholologists. In non-biopsied cases, the clinical and High Resolution Computed Tomography (HRCT) evaluations are, on average, equally influential. Key clinical information includes the identification of a cause or relevant underlying systemic disease, and the assimilation of serial data allowing definition of the natural history or treated course of disease. In patients undergoing surgical biopsy, clinical and HRCT findings are usually inconclusive and the histological features tend to carry the most diagnostic weight. However, it should be expected that the final diagnosis will differ from the histological diagnosis in a significant minority of patients, when all available information is integrated.
\end{abstract}

Keywords Multidisciplinary diagnosis · Aetiology $\cdot$ Natural history $\cdot$ High resolution computed tomography $\cdot$ Diagnostic surgical biopsy · Pulmonary function tests · Bronchoscopy · Bronchoalveolar lavage $\cdot$ Transbronchial lung biopsy $\cdot$ Chest radiography · Autoimmune serology · Pulmonary fibrosis . Interstitial lung disease $\cdot$ Diagnosis $\cdot$ Multidisciplinary approach $\cdot$ HRCT $\cdot$ Surgical lung biopsy

\footnotetext{
A. Gogali $(\bowtie) \cdot$ A. U. Wells

Interstitial Lung Disease Unit, Royal Brompton Hospital, C/O Emmanuel Kaye Building,

Manresa Rd, Chelsea, London SW3 6LR, UK

e-mail: athenagogali@yahoo.com

A. U. Wells

e-mail: athol.wells@rbht.nhs.uk
}

\section{Introduction}

Interstitial lung disease (ILD) is a broad term that includes a variety of diverse entities with overlapping histopathologic patterns of inflammation and fibrosis that affect not only the interstitium, but also the alveolar spaces and, in many cases, the acinar regions. Although these disorders have different pathogeneses and etiologies, which in many instances are unknown, they were grouped historically, due to similar clinical and chest radiographic presentations. Nevertheless, many secondary infiltrative abnormalities, always considered in the formulation of a differential diagnosis, such as infection, pulmonary oedema and malignancy, are conventionally excluded, whereas others, such as pulmonary involvement in connective tissue disease or occupational lung disease, are retained in most classifications (Table 1).

Idiopathic disease poses particular difficulties. In 2002, the American Thoracic Society/European Respiratory Society (ATS/ERS) international consensus committee reclassification of idiopathic interstitial pneumonias (IIPs) provided a framework for the separation of a number of disease entities with diverse natural histories and treated outcomes [1]. Non-specific interstitial pneumonia (NSIP) was acknowledged as a new entity, distinct from idiopathic pulmonary fibrosis (IPF). This reclassification made new demands on the accuracy of diagnosis in clinical practice.

Historically, histopathologic evaluation has been viewed as the gold standard for diagnosis in interstitial lung disease. That perception has changed. It is now widely accepted that all clinical, radiological, and, when available, histopathological data should be integrated into the final diagnosis $[2,3 \cdot \bullet, 4]$, in the context of a multidisciplinary approach. The limitations of a histological diagnosis, apart from the difficulty in many cases of performing a diagnostic surgical biopsy, are now better understood. 
Table 1 Diffuse parenchymal lung disease

Associated with systemic diseases

Rheumatological:

Systemic sclerosis, rheumatoid arthritis, polymyositis/ dermatomyositis, systemic lupus erythematosus, Sjögren's syndrome, ankylosing spondylitis

Vasculitis:

Wegener's granulomatosis, Churg-Strauss granulomatosis, microscopic polyangiitis, pulmonary-renal syndrome (including Goodpasture's syndrome), capillaritis, Behçet's syndrome

Vascular:

Primary pulmonary hypertension, idiopathic pulmonary haemosiderosis, pulmonary veno-occlusive disease, antiphospholipid syndrome

Diseases caused by environmental triggers or drug ingestion Hypersensitivity pneumonitis: fungal, bacterial, avian, chemical Fibrogenic inorganic dusts: asbestosis, silica, hard metal alloyberyllium, coal, aluminium

Therapeutic agents (e.g. bleomycin, amiodarone), illicit drugs, radiation, pesticides, oxygen and other inhaled gases

Granulomatous diseases

Sarcoidosis, hypersensitivity pneumonitis, berylliosis, Langerhans cell histiocytosis, Wegener's granulomatosis, Churg Strauss syndrome, lymphomatoid granulomatosis, bronchocentric granulomatosis

Idiopathic interstitial pneumonias

Idiopathic pulmonary fibrosis, non-specific interstitial pneumonia, desquamative interstitial pneumonia, respiratory bronchiolitisinterstitial lung disease, acute interstitial pneumonia, cryptogenic organizing pneumonia, lymphocytic interstitial pneumonia

Other diffuse lung diseases

Inherited disorders: tuberous sclerosis, neurofibromatosis , Hermansky-Pudlak syndrome, lipid storage disorders, familial idiopathic pulmonary fibrosis

Pulmonary eosinophilia: known causes (fungi, parasites, drugs), acute idiopathic, chronic idiopathic

Lymphangioleiomyomatosis

Alveolar proteinosis

Alveolar microlithiasis

Amyloidosis

Chronic aspiration

In routine practice, diagnosis is sometimes uncertain despite intensive investigation, and it is often difficult to decide the length to which a specific diagnosis should be pursued. Thus a simplified pragmatic approach to diagnosis is essential. This approach includes careful clinical evaluation and a logical sequence of tests:

1. clinical history

2. clinical examination

3. chest radiography

4. pulmonary function tests

5. selective blood tests

6. high resolution computed tomography
7. bronchoscopy

8. surgical biopsy

\section{Clinical history}

Most patients present with insidious dyspnoea, frequently accompanied by dry cough. An acute presentation with apparently idiopathic disease narrows the differential diagnosis to acute interstitial pneumonia, diffuse alveolar haemorrage, fulminant organising pneumonia, acute pneumonitis, acute pulmonary eosinophilia, hypersensitivity pneumonitis (HP) and drug-induced ILD.

Wheeze is much rarer, and reveals the presence of an airway-centred component. It is sometimes prominent in HP, sarcoidosis, lymphangioleiomyomatosis and Langerhans cell histiocytosis (LCH). Pleuritic chest pain often occurs in rheumatological diseases and occasionally in drug-induced disease, while pneumothorax should prompt suspicion of cystic lung disease, especially LCH and lymphangioleiomyomatosis. Haemoptysis may be indicative of the existence but not the severity of diffuse alveolar haemorrhage due to capillaritis. Systematic symptoms as fever, malaise, fatigue, arthralgias may be present in sarcoidosis and rheumatological disease. Specific symptoms as Raynaud's phenomenon, sicca syndrome, rash or reflux should be carefully searched.

A history of cigarette smoking identifies a predisposition to LCH, Desquamative Interstitial Pneumonia (DIP) and Respiratory Bronchiolitis ILD [5]. Paradoxically, current smoking is rare in sarcoidosis and HP.

The previous medical history may provide crucial information, including diagnoses of rheumatological disease or disorders that mimic ILD like malignancy or heart failure. A detailed list of medications should be always asked so as to eliminate the possibility of drug-induced lung disease [6, 7]. Detailed occupational history [8, 9] and environmental exposure may reveal asebstos exposure (railway rolling-stock construction, shipyard workers, power station construction and maintenance workers, naval boilermen, garage workers, workers in the building trade), pneumoconiosies or inhalation of organic dusts [10]. Other relevant historical information includes foreign travel, which may raise the possibility of parasitic infection as an explanation of pulmonary eosinophilia.

A great deal of information can often be obtained on the longitudinal behaviour of disease from the evolution of symptoms, serial chest radiographic data, and, less frequently, serial spirometric volumes.

\section{Clinical examination}

On auscultation, predominantly basal fine end inspiratory crackles are a cardinal feature of pulmonary fibrosis and are 
usually present in IPF and NSIP, occurring sporadically in a number of other ILDs. Expiratory wheeze is rare and indicative of airway disease. Inspiratory squawks are a characteristic finding in HP and obliterative bronchiolitis.

Digital clubbing is uncommon in ILDs except in IPF (approximately $50 \%$ of the patients), idiopathic NSIP and asbestosis. Rarely, it may be seen in HP and sarcoidosis. In advanced disease, clinical evidence of secondary pulmonary hypertension (e.g. pedal oedema) may be present.

Systemic examination should be never omitted and may reveal ocular disease (in sarcoidosis or vasculitis), skin disease (in sarcoidosis or rheumatological disease), musculoskeletal signs (in rheumatological disease) and neurological abnormalities (in sarcoidosisis, rheumatological disease and vasculitis) [11].

\section{Chest radiography}

Although, High Resolution Computed Tomography (HRCT) has now supplanted chest radiography in routine diagnosis, the chest radiograph continues to provide useful information. It discloses:

a. The size of the lungs: fibrosing lung diseases tend to have reduced lung volumes. Normal sized or large lungs on chest radiography occur in coexistence of emphysema and pulmonary fibrosis, in $\mathrm{LCH}$ and lymphangioleiomyomatosis.

b. The distribution of disease: Primary fibrosing disorders including IPF, fibrotic NSIP, pulmonary fibrosis in rheumatological disease and asbestosis, produce predominantly basal reticular or reticulonodular abnormalities, which may also be overtly peripheral when disease is not advanced. By contrast, granulomatous disorders most often have a predominantly upper and mid zone distribution.

c. The type of abnormalities: Linear pattern (fibrosing lung disease), nodules ( $<1 \mathrm{~mm}$ : miliary $\mathrm{TB}$, haemosidirosis, $<5 \mathrm{~mm}$ : sarcoidosis, silicosis, $>5 \mathrm{~mm}$ : rheumatoid arthritis, Wegener, lymphoma, malignancy), infiltrations (Organizing pneumonia, pulmonary eosinophilia, haemorrhage, infection, bronchoalveolar carcinoma).

d. Pleural thickening, with or without effusion, which may occur in rheumatoid arthritis, systemic lupus erythematosus, vasculitis, asbestosis and drug-induced lung disease.

e. Lymphadenopathy: Symmetrical hilar lymphadenopathy is usually indicative of sarcoidosis, but tuberculosis, lymphoma and other malignancies should always be considered, especially if the changes are unilateral.

In the correct clinical setting, chest radiographic findings often suffice for a confident diagnosis of sarcoidosis (predominantly upper zone fibrotic change, variably associated with lymphadenopathy and hilar retraction towards the apices). However, in other patients presenting with an undiagnosed ILD, HRCT is warranted. Serial chest radiographs often add invaluable information on the duration and the natural history of the disease.

\section{Pulmonary function tests}

The cardinal pattern of most ILDs is a restrictive pulmonary defect, reduced gas transfer (DLco) and hypoxia, at rest or on exercise [12]. Thus, the contribution of pulmonary function tests to an ILD diagnostic algorithm is usually minor. The exception is the presence of prominent airflow obstruction that may be indicative of a small number of ILDs including LCH, lymphangioleiomyomatosis, intrinsic airways disease (i.e. bronchiolitis) and sarcoidosis. In smokers with airflow obstruction, concurrent emphysema is an important consideration. Preservation of DLco is indicative of intrinsic airway disease and discriminates usefully between bronchiolitis and emphysema [13], but this distinction is not useful if there is admixed interstitial lung disease. In the absence of emphysema, a mixed ventilatory defect usually denotes an airway-centred disease component, increasing the likelihood of a diagnosis of HP, sarcoidosis or connective tissue disease (as opposed to idiopathic interstitial pneumonia). The admixture of emphysema and fibrosis (most commonly IPF or fibrotic NSIP) also produces a mixed pattern, but with the distinctive profile of relative preservation of lung volumes in association with severe reduction in DLco levels [14]. A reduction in gas transfer disproportionate to lung volumes may also be indicative of concurrent pulmonary vascular disease [15], especially when accompanied by severe resting hypoxia or major arterial oxygen desaturation with minor exertion. In sharp contrast, DLco and Kco levels 200-300 \% above the upper limit of normal, measured using single breath testing, are characteristic features of recent diffuse alveolar hemorrhage syndromes [16]. In the absence of alveolar haemorrhage, a restrictive ventilatory defect combined with a major increase in Kco is a reliable indicator of extra-pulmonary restriction [17]. The wide variety of patterns of pulmonary function impairment reflects the heterogeneous pathological processes in the ILDs, but in the HRCT era, patterns of pulmonary function impairment usually add little to diagnostic evaluation.

\section{Blood tests}

Routine haematology and biochemical tests have little diagnostic value in ILDs with the exception of a peripheral blood eosinophilia, which may be indicative of eosinophilic lung disease. Elevated liver functions or renal impairment may suggest a multi-organ process 
(e.g. sarcoidosis, vasculitis). Increased levels of angiotensin converting enzymes are a helpful ancillary diagnostic finding in some patients with sarcoidosis, although not pathognomonic and not always present. Routine immunoglobulin estimation may disclose hypogammaglobulinaemia in undiagnosed granulomatous disorders.

Autoantibody testing is an important part of routine evaluation. ESR, ANA, RF, SCL-70, anti-t-RNA synthetase autoantibodies, SS-A, SS-B, anti-RNP autoantibody and ANCA may disclose occult rheumatological disease. Low titres of ANA and RF are commonly found in IPF and idiopathic fibrotic NSIP but have no clinical significance [18].

Panels of specific precipitins to organic antigens may point to an exact etiology in HP. However, positive precipitins confirm only exposure and are often present in healthy individuals (e.g. healthy pigeon breeders). More importantly, the absence of precipitins does not exclude a diagnosis of HP.

\section{High Resolution Computed Tomography}

HRCT has been the most important diagnostic advance in interstitial lung disease in the last two decades. The superior sensitivity and specificity of HRCT, compared to chest radiography, is well recognized, and several studies have confirmed its diagnostic accuracy against histological findings in specific clinical contexts $[19,20 \bullet]$. HRCT may identify diffuse parenchymal disease undetectable on plain $\mathrm{x}$-ray. In one large series, approximately $10 \%$ of patients with biopsy-proven diffuse lung disease had an apparently normal chest radiograph [21]. In some cases, appearances on HRCT are pathognomonic of a histo-specific diagnosis: typical HRCT patterns are listed in Table 2. In other cases, HRCT appearances are not diagnostic in isolation but give rise to a secure non-invasive diagnosis when integrated with clinical information.

A key step in the diagnostic process is to discriminate between predominantly inflammatory and predominantly fibrotic disease. Anatomic distortion and reticular abnormalities on HRCT are strongly indicative of irreversible fibrotic disease and honeycomb change reflects end stage fibrosis. Groundglass attenuation was once thought to represent active inflammation, but this is generally true only in the absence of admixed reticular abnormalities or traction bronchiectasis. Thus, ground glass is often indicative of fine fibrosis, especially in cases of fibrotic NSIP or sarcoidosis. Consolidation is easier to interpret as it is usually inflammatory and reversible, but dense fibrosis can occasionally result in "fibrotic consolidation", especially in sarcoidosis.

If disease is fibrotic, as in the large majority of cases, the next important question is whether HRCT findings are typical of IPF. As stated in the recent ATS/ERS recommendations, IPF can be diagnosed confidently on HRCT when
Table 2 High Resolution Computed Tomography features recognized as typical of selected diffuse lung diseases

Idiopathic pulmonary fibrosis: Lower zone, subpleural predominance, maximal posterobasally, predominantly reticular pattern with associated honeycombing.

Nonspecific interstitial pneumonia: Two frequent appearances. The variant that overlaps with idiopathic pulmonary fibrosis in disease distribution: ground-glass attenuation predominates, with a variable fine reticular component and traction bronchiectasis, but no honey combing. The variant that overlaps with cryptogenic organizing pneumonia: consolidation with surrounding ground-glass attenuation and a variable fine reticular pattern.

Desquamative interstitial pneumonia: Ground-glass attenuation, sometimes diffuse, sometimes basal and peripheral centered, frequent associated fibrotic cysts with anatomic distortion and traction bronchiectasis.

Acute interstitial pneumonia: Widespread ground-glass attenuation admixed with features of fibrosis, usually with airspace consolidation, and occasional emphysema.

Respiratory bronchiolitis - interstitial lung disease: Patchy ground-glass attenuation, poorly defined centrilobular nodules, occasional mosaic attenuation, prominent bronchial wall thickening.

Sarcoidosis: Highly variable. Nodules distributed along bronchovascular bundles, interlobular septa, and subpleurally including the fissure; ground-glass attenuation that may represent either inflammation or fine fibrosis; reticular abnormalities, representing fibrosis; distortion most commonly occurring in upper zones with posterior displacement of the upper lobe bronchus; air trapping; associated hilar and mediastinal lymphadenopathy.

Subacute hypersensitivity pneumonitis: Widespread ground-glass attenuation, often containing poorly defined centrilobular nodules, admixed with areas of black lung (mosaic attenuation), representing air trapping and enhanced on expiratory high-resolution computed tomography.

Cryptogenic organizing pneumonia: Bilateral patchy consolidation, subpleural and predominantly basal in the majority, occasional peribronchial distribution, associated often sparse nodules up to $1 \mathrm{~cm}$ in diameter.

Constrictive bronchiolitis: Patchy areas of hyperlucency enhanced on expiration, which may not change in cross-sectional diameter on full expiration; associated bronchiectasis and bronchial wall thickening.

Langerhans cell histiocytosis: Bizarre cyst shapes and associated nodules throughout the lung fields but sparing the costophrenic angles and tips of the lingula and middle lobes. Associated emphysema is common.

Pulmonary lymphangioleiomyomatosis: Homogeneously distributed, thin-walled parenchymal cysts, varying from a few millimeters to several centimeters in diameter; associated with retrocrural adenopathy, pleural effusion, thoracic duct dilatation, pericardial effusion, and pneumothorax.

there is honeycombing with little ground-glass attenuation in a predominantly basal and sub-pleural distribution. It is now known that HRCT appearances considered typical of IPF by experienced thoracic radiologists have a positive predictive value of over $95 \%$ [22]. In fact, honeycombing appears to be the most useful HRCT feature to differentiate histologic usual interstitial pneumonia (UIP) from either 
cellular or fibrotic NSIP (odds ratios 5.16 and 2.1, respectively) [23]. Unfortunately, this 'typical' UIP pattern on HRCT is only present in 40-60\% of the patients with IPF [24••, 25]. Additionally, inter-observer agreement for diagnosing a pattern of UIP on HRCT has been reported to be moderate (kappa 0.40-0.55) [26-28]. There was even lower agreement between community-based and academic radiologists (kappa 0.11-0.34). Community-based physicians were more likely to assign a diagnosis of UIP/IPF, compared with academic-based physicians [28].

If appearances are not typical of IPF, the five most important differential diagnoses of apparently idiopathic disease (based upon their prevalence in routine practice) are IPF with atypical HRCT appearances, sarcoidosis, NSIP, HP (with the antigen unknown) and the fibrotic sequelae of cryptogenic organizing pneumonia (COP). Atypical IPF is especially likely when HRCT appearances are not typical of any of the other four disorders, but it is also frequently resembles fibrotic NSIP. In large series of pathologically proven UIP [24••, 29], an HRCT pattern of definite UIP was seen in only one third of the cases, whereas more than one third had HRCT appearances suggestive of NSIP. Radiologic diagnosis of NSIP is a challenge, as when experienced radiologists are highly confident that their radiographic diagnosis of NSIP identified underlying histologic NSIP, they are correct $73 \%$ of the time [30]. The absence of honeycombing as the predominating HRCT feature (in the eyes of experienced radiologists) for histologic NSIP has a negative predictive value of $90 \%$ [30]. The presence of concurrent emphysema obscures further the distinction between UIP and NSIP on HRCT [31•].

In summary, the combination of clinical and HRCT information provides a confident first choice diagnosis in the majority of ILD patients, and significantly shortens the differential diagnosis in many other cases. In a minority of patients, when the CT appearances are inconclusive, or when important management questions are raised and the distinction between two or three disorders is essential, a diagnostic surgical biopsy is required.

\section{Bronchoscopy}

This semi-invasive procedure provides the opportunity to perform bronchoalveolar lavage (BAL) and endobronchial/ transbronchial biopsies. In the 1980 s, BAL was viewed as a crucial step in the diagnostic procedure, but after the introduction of HRCT, its value has been downgraded. Nevertheless, bronchoalveolar lavage can be diagnostic in some disorders, including alveolar proteinosis (milky effluent; PAS-positive material), LCH (increased numbers of Langerhans cells identified by CD1a staining $>4 \%$ ), eosinophilic pneumonia (eosinophils $>25 \%$ ), alveolar haemorrhage (iron-laden macrophages), and exposure to hard metals (metal fibres enclosed in multinuclear giant cells). Additionally, diseases that mimic ILDs as infections in immunocompromised (Pn. Carinii, fungi, CMV, m.Tb), bronchoalveolar carcinoma, diffuse carcinomatosis are disclosed by BAL. In other cases, BAL has only an ancillary diagnostic role.

The presence of a prominent BAL lymphocytosis should increase the suspicion that a fibrosing process may be due to HP or sarcoidosis, and may, in itself, justify a surgical biopsy in some cases. A prominent BAL lymphocytosis may also be present in COP, cellular NSIP, lymphoid interstitial pneumonia (LIP), drug toxicity or non-LIP lymphoproliferative disorders. Furthermore, an elevated CD4+/ CD8+ ratio of 3.5 is relatively specific for active sarcoidosis $[32,33]$, but the sensitivity is low and the ratio increases in elderly patients [34]. Thus, use of the CD4+/CD8+ ratio as a routine component of BAL analysis is questionable and may increase the cost of the procedure considerably.

In the idiopathic interstitial pneumonias, the role of BAL remains contentious. The 2002 ATS/ERS recommendations for the diagnosis of IPF [1] specified that compatible BAL findings (i.e. the absence of lymphocytosis) were a requirement for the non-invasive diagnosis of IPF. By contrast, the recent guideline from The joint ATS/ERS/JRS/ALAT [35••] states that the presence of the described UIP pattern on HRCT imaging is sufficient to make a diagnosis of IPF in the appropriate clinical setting, and eliminates the need for BAL cellular analyses. However, in a recent study [36••], $8 \%$ of patients with a confident clinical and HRCT diagnosis of IPF had a BAL lymphocytosis of $\geq 30 \%$, and in these cases an alternative diagnosis of NSIP or HP was made, validated at biopsy or by subsequent disease behavior. Based on these data and on personal clinical experience, some clinicians do not accept the recommendation that BAL not be performed in the setting of a confident diagnosis of IPF. Even accepting that this issue is not resolved, in cases of suspected IPF, with HP or NSIP realistic differential diagnoses, BAL findings may strongly influence the threshold for performing a diagnostic surgical biopsy.

Endobronchial and transbronchial (TBLB) biopsies provide only a small volume of bronchial and peribronchial tissue. The reported diagnostic yield of TBLB varies between $27 \%$ and $79 \%$ [37]. This wide range reflects the multiplicity of factors influencing the yield of the procedure, including the distribution of the lesion (focal or diffuse), status of the immune system of the patient, small size of the obtained samples, confounding due to crush artifact and failure to penetrate beyond the peribronchial sheath [38, 39]. TBLB is most helpful in diseases with a bronchocentric distribution, especially sarcoidosis and lymphangitis carcinomatosis. Occasionally, transbronchial biopsy findings can help to cement a diagnosis of HP or organizing pneumonia, when intergrated with clinical/radiological information (including BAL). Bronchoscopic biopsy procedures have little 
diagnostic value in IIPs other than COP [40]. Recently, it has been proposed that the use of larger forceps via rigid bronchoscope would increase the diagnostic yield of TBLB and avoid crush artefacts [41]. However, in successful cases, the underlying diagnoses were forms of inflammatory ILD, whereas in undiagnosed cases, the most frequent diagnosis at surgical biopsy was UIP/IPF, followed by fibrotic NSIP and HP. Thus, although undoubtedly promising, this method is yet to be validated in the diagnosis of IPF or the other IIPs.

\section{Surgical biopsy}

Surgical biopsy, formerly performed as an open lung procedure but now widely obtained using video assisted thoracoscopic surgery (VATS), is the last step in the diagnostic ILD algorithm, which adds invaluable information and diagnostic accuracy. Histopathologic assessment was, until recently, regarded as the diagnostic "gold standard" in interstitial lung disease, because it provided the most accurate prediction of the natural history and treated course of disease. Nevertheless, the application of HRCT has reduced the performance of diagnostic surgical biopsies, even in referral centres. Moreover, it has become increasingly clear that surgical biopsy is a useful diagnostic tool with limitations. Firstly, not all patients are fit to undergo a surgical procedure due to comorbidities or inadequate pulmonary reserve. In one series, patients with relatively advanced IPF (mean gas transfer level of $<35 \%$ of predicted) had a mortality ascribable to biopsy of $15 \%$ [42]. Thus, the threshold for performing a diagnostic surgical biopsy should rise in severe disease. It is the view of the authors that if the DLco level is $<30 \%$, a diagnostic surgical biopsy should be performed only if central to accurate management.

"Sampling error" is a second important limitation that has been documented by more than one research team [43, 44]. It is known that in IPF, the histologic patterns of UIP and NSIP may co-exist [44]. If the "wrong area" is sampled, a histopathologic diagnosis of NSIP can sometimes be misleading, because the disease course may reflect the presence of UIP in non-sampled areas. Similarly, sampling only of end-stage fibrosis areas may not add much to diagnostic precision. The acquisition of biopsies from multiple lobar sites, though, guided by HRCT, increases the likelihood of obtaining representative tissue.

Additionally, significant interobserver variation exists, even between experienced pathologists. In the largest study of interobserver variation between pathologists, agreement was only moderate, with the first choice diagnosis assigned with low confidence in over $20 \%$ of biopsies [45]. This observation is not especially surprising, as in some cases, histological appearances may be intermediate between two patterns, leading to appropriate disagreement between observers as to the first choice diagnosis.
Surgical biopsy is increasingly reserved for cases in which clinical or HRCT information is inconclusive or discordant and a confident management strategy cannot be constructed based on non-invasive data.

\section{Integrated diagnosis in ILD: key issues}

Multidisciplinary approach

The first and most important step is to collect and integrate all the above information. An overemphasis on histological data at the expense of HRCT and clinical information may result in seriously flawed diagnostic conclusions. A specific histological pattern may be seen in the context of different clinical entities, and the final diagnosis should be made in relation to the clinical/radiological setting. For example, UIP is the characteristic histological pattern in IPF, but also occurs in rheumatological disorders, drug-induced lung disease and HP [46••]. Similarly, in an ATS sponsored NSIP workshop, in which a large number of cases were reviewed, a multidisciplinary diagnosis other than idiopathic NSIP (usually HP, IPF or COP) was made in over half of cases submitted to the workshop with a confirmed histologic pattern of NSIP. HRCT findings were the most common reason for a diagnosis other than idiopathic NSIP, followed by a combination of HRCT and clinical data [4].

Flaherty et al. have highlighted the value of multidisciplinary approach in ILDs [2]. In the case of IPF, when a confident pre-biopsy diagnosis was made by clinicians or radiologists, the diagnosis virtually never changed with the addition of histological data. By contrast, in the remaining cases, diagnoses made by clinicians and radiologists changed in approximately $50 \%$ of cases when biopsy data were considered. However, it should also be stressed that the final diagnosis differed from the histological diagnosis in over $25 \%$ of cases. This provided support for the view that although histology has a major beneficial impact on diagnostic confidence, the diagnostic "gold standard" is consensus decision making [28].

\section{When to perform a biopsy}

The most difficult dilemma for the clinician is whether to recommend a surgical biopsy or to accept a degree of diagnostic uncertainty. There are no guidelines on this question, and every case should be considered individually. Usually biopsy is reserved for cases in which clinical or HRCT information is inconclusive or discordant. As a general rule, a surgical biopsy should always be pursued in patients fit for these procedures (as judged by age, disease severity and comorbidity) if a confident management strategy cannot be constructed from non-invasive evaluation. In 
that context, a diagnostic biopsy will usually allow a confident multidisciplinary diagnosis to be made and, thus, the construction of a logical management plan. However, if the exact diagnosis is uncertain but the pattern of disease behaviour is already clear, a diagnostic biopsy is much less likely to alter management. For example, when it is already known from previous investigations that fibrotic abnormalities are long-standing and wholly stable, the pursuit of histological diagnosis is unlikely to change management. Broadly, longitudinal disease behaviour in ILD can be subdivided into five patterns based upon underlying cause (if any), morphological assessment using HRCT and observed change over time (previous chest radiographs, symptoms and, occasionally, pulmonary function tests):

1. Self-limited inflammation, usually caused by an extrinsic agent (as in drug induced disease, HP and RBILD) but may also be idiopathic as in a subset of patients with sarcoidosis.

2. Stable fibrotic disease (e.g. sarcoidosis, patients with formerly active rheumatological disorders).

3. Major inflammation, with or without supervening fibrosis (e.g. drug-induced lung disease; COP; DIP; HP; sarcoidosis; aggressive inflammatory disease in rheumatological disease).

4. Slowly progressive fibrotic disease (e.g. sarcoidosis; $\mathrm{HP}$; rheumatological conditions; many patients with fibrotic NSIP).

5. Inexorably progressive fibrotic disease despite treatment (e,g. IPF, but an IPF-like course is sometimes observed in idiopathic fibrotic NSIP, rheumatological disease and in a subset of patients with chronic HP.)

A histological diagnosis is required more frequently in apparently idiopathic disease in order to discriminate inherently stable from potentially progressive fibrotic disease, and major inflammation (with a high risk of evolution to fibrosis with under-treatment) from inexorably progressive fibrotic disease, because therapeutic intervention differs substantially. If a confident management strategy, based upon disease behaviour, can be constructed from non-invasive evaluation, surgical biopsy is less likely to be warranted. For example, mortality is very similar in IPF and fibrotic NSIP when DL CO levels are less than $35 \%$, despite striking differences in survival when disease is less severe [47]. Thus, for younger patients (under the age of 60) presenting with a typical clinical picture of severe/end-stage IPF and HRCT features suggestive of fibrotic NSIP, immediate referral for consideration of lung transplantation is warranted, without the need for a high risk diagnostic surgical procedure.

Discussion with the patient

Lastly, it is extremely important that a detailed, informative discussion with the patient should precede any decision on whether to perform a biopsy. The involvement of the patient in this decision is seldom discussed but is, we believe, pivotal. In some cases, a biopsy is essential for accurate management and the role of the physician in discussion is to make this clear to the patient. However, in a larger proportion of cases, physician recommendations on the need for biopsy are more nuanced. Often, a biopsy appears warranted, but an approximate management strategy, although recommended with less precision and confidence, can be devised without biopsy information. Sometimes decisions on the need for biopsy are genuinely a "50/50 call". The views of the patient should always be sought and if biopsy decisions are indeed a close call, the preferences of the patient should determine the final decision. However, it is important to stress that some patients prefer biopsy decisions to be made by their physician, do not wish to state a preference and should not be compelled to do so.

\section{Conclusion}

The diagnosis of ILDs is a difficult task and a systematic diagnostic algorithm, based upon careful clinical evaluation and a logical sequence of tests, is essential. It has to be stressed that there is no single "gold standard" for diagnosis in ILDs, merely the "silver standards" of clinical, radiologic, and histopathologic evaluation. An integrated approach is essential as the final multidisciplinary diagnosis differs from the clinical, HRCT and histological diagnoses in a significant minority of patients.

Disclosure No potential conflicts of interest relevant to this article were reported.

\section{References}

Papers of particular interest, published recently, have been highlighted as:

- Of importance

- Of major importance

1. Joint American Thoracic Society and European Respiratory Society Group. International multidisciplinary consensus classification of the idiopathic interstitial pneumonias. Am J Respir Crit Care Med. 2002;165:277-304.

2. Flaherty KR, King Jr TE, Raghu G, et al. Idiopathic interstitial pneumonia: what is the effect of a multidisciplinary approach to diagnosis? Am J Respir Crit Care Med. 2004;170:904-10.

3. •- Schmidt SL, Sundaram B, Flaherty KR. Diagnosing fibrotic lung disease: when is high-resolution computed tomography sufficient to make a diagnosis of idiopathic pulmonary fibrosis? Respirology. 2009;14:934-9. A perceptive review on the contribution of HRCT to the diagnosis of IPF. This article offers clinicians useful insights on the integration of HRCT findings into the multidisciplinary approach. 
4. Travis WD, Hunninghake G, King Jr TE, et al. Idiopathic nonspecific interstitial pneumonia: report of an American thoracic society project. Am J Respir Crit Care Med. 2008;177:1338-47.

5. Caminati A, Harari S. Smoking-related interstitial pneumonias and pulmonary Langerhans cell histiocytosis. Proc Am Thorac Soc. 2006;3:299-306.

6. Camus P, Bonniaud P, Fanton A, et al. Drug induced and iatrogenic infiltrative lung disease. Clin Chest Med. 2004;25:479-519.

7. www.pneumotox.com

8. Glazer CS, Newman LS. Occupational interstitial lung disease. Clin Chest Med. 2004;25:467-78.

9. Neurnberg AM, Christiani DC. The future of occupationally related diffuse lung disease. Semin Respir Crit Care Med. 2008;29:680-4.

10. Hanak V, Golbin JM, Ryu JH, et al. Causes and presenting features in 85 consecutive patients with hypersensitivity pneumonitis. Mayo Clin Proc. 2007;82:812-6.

11. Tzelespis GE, Toya SP, Moustopoulos HM. Occult connective tissue diseases mimicking idiopathic interstitial pneumonias. Eur Respir J. 2008;31:11-20.

12. Gibson GJ. Clinical tests of respiratory function. London: Chappell and Hall; 1996. p. 223-4.

13. Hansell DM, Rubens MB, Padley SPG, et al. Obliterative bronchiolitis: individual CT signs of small airways disease and functional correlation. Radiology. 1997;203:721-6.

14. Gould GA, Redpath AT, Ryan M, et al. Lung CT density correlates with measurements of airflow limitation and the diffusing capacity. Eur Respir J. 1991;4:141-6.

15. Burke CM, Glanville AR, Morris AJR, et al. Pulmonary function in advanced pulmonary hypertension. Thorax. 1987;42:151-5.

16. Lipscomb DJ, Patel K, Hughes JM. Interpretation of increases in the transfer coefficient for carbon monoxide (TLCO/VA or KCO). Thorax. 1978;33:728-33.

17. Wright PH, Hansen A, Kreel L, et al. Respiratory function changes after asbestos pleurisy. Thorax. 1980;35:31-6.

18. Turner-Warwick M, Burrows B, Johnson A. Cryptogenic fibrosing alveolitisclinical features and their influence on survival. Thorax. 1980;35:171-80.

19. Johkoh T, Muller NL, Cartier Y, et al. Idiopathic interstitial pneumonias: diagnostic accuracy of thin-section CT in 129 patients. Radiology. 1999;211:555.

20. - Quadrelli S, Molinari L, Ciallella L, et al. Radiological versus histopathological diagnosis of usual interstitial pneumonia in the clinical practice: does it have any survival difference? Respiration. 2010;79:32-7. This study addresses the possibility that IPF patients diagnosed by means of a surgical biopsy, usually without honeycombing, might have a different outcome phenotype. However, the authors report that survival was the same in IPF patients diagnosed using HRCT and IPF patients diagnosed at surgical biopsy (presumably with atypical HRCT appearances).

21. Epler GR, McLoud TC, Gaensler EA, et al. Normal chest radiographs in chronic diffuse infiltrative lung disease. N Engl J Med. 1978;298:934-9.

22. Hunninghake GW, Zimmerman MB, Schwartz DA, et al. Utility of a lung biopsy for the diagnosis of idiopathic pulmonary fibrosis. Am J Respir Crit Care Med. 2001;164:193-6.

23. Sumikawa H, Johkoh T, Ichikado K, et al. Usual interstitial pneumonia and chronic idiopathic interstitial pneumonia: analysis of CT appearance in 92 patients. Radiology. 2006;241:258-66.

24. •- Sverzellati N, Wells AU, Tomassetti S, et al. Biopsy-proved idiopathic pulmonary fibrosis: spectrum of non diagnostic thinsection CT diagnoses. Radiology. 2010;254:957-64. In this study, reported from institutions in which atypical HRCT appearances were a major indication for a diagnostic surgical biopsy, the range of HRCT findings was described. Notably, survival did not differ in biopsy-proven IPF according to whether HRCT appearances were typical or atypical.
25. Sverzellati N, De Filippo M, Bartalena T, et al. High-resolution computed tomography in the diagnosis and follow-up of idiopathic pulmonary fibrosis. Radiol Med. 2010;115:526-38.

26. Thomeer M, Demedts M, Behr J, et al. Multidisciplinary interobserver agreement in the diagnosis of idiopathic pulmonary fibrosis. Eur Respir J. 2008;31:585-91.

27. Aziz ZA, Wells AU, Hansell DM, et al. HRCT diagnosis of diffuse parenchymal lung disease: inter-observer variation. Thorax. 2004;59:506-11.

28. Flaherty KR, Andrei AC, King Jr TE, et al. Idiopathic interstitial pneumonia: do community and academic physicians agree on diagnosis? Am J Respir Crit Care Med. 2007;175:1054-60.

29. MacDonald SL, Rubens MB, Hansell DM, et al. Nonspecific interstitial pneumonia and usual interstitial pneumonia: comparative appearances at and diagnostic accuracy of thin-section CT. Radiology. 2001;221:600-5.

30. Elliot TL, Lynch DA, Newell Jr JD, et al. High-resolution computed tomography features of nonspecific interstitial pneumonia and usual interstitial pneumonia. J Comput Assist Tomogr. 2005;29:339-45.

31. - Akira M, Inoue Y, Kitaichi M, et al. Usual interstitial pneumonia and nonspecific interstitial pneumonia with and without concurrent emphysema: thin-section CT findings. Radiology. 2009;251:2719. This study highlights the difficulties that can arise in categorizing HRCT findings as typical of IPF when there is concurrent smoking-related emphysema. This is a frequent problem in routine diagnosis as emphysema is present on HRCT in up to $40 \%$ of IPF patients.

32. Costabel U, Zaiss AW, Guzman J. Sensitivity and specificity of BAL findings in sarcoidosis. Sarcoidosis. 1992;9:211-4.

33. Winterbauer RH, Lammert J, Selland M, et al. Bronchoalveolar lavage cell populations in the diagnosis of sarcoidosis. Chest. 1993;104:252-61.

34. Meyer KC, Soergel P. Bronchoalveolar lymphocyte phenotypes change in the normal aging human lung. Thorax. 1999;54:697-700.

35. •• Raghu G, Collard HR, Egan JJ, et al. An official ATS/ERS/JRS/ ALAT statement: idiopathic pulmonary fibrosis: evidence-based guidelines for diagnosis and management. Am J Respir Crit Care Med. 2011;183:788-824. Impressive distillation of the literature with formulation of guidelines for the diagnosis and treatment of IPF: a tour de force as a summary of current data. As with all guideline statements made by experts, some recommendations have caused difficulties when applied by less experienced practitioners and need to be reappraised before long.

36. •• Ohshimo S, Bonella F, Cui A, et al. Significance of bronchoalveolar lavage for the diagnosis of idiopathic pulmonary fibrosis. Am J Respir Crit Care Med. 2009;179:1043-7. An important observation that, if reproduced, should stimulate reappraisal of the diagnostic role of BAL. Recent expert groups have recommended against routine BAL when the clinical and HRCT features are typical of IPF. These authors showed that in a small patient subgroup, a BAL lymphocytosis of over $30 \%$ was reliably indicative of an alternative diagnosis, validated ay biopsy or by subsequent disease behaviour.

37. Bradley B, Branley HM, Egan JJ, et al. Interstitial lung disease guideline: the British thoracic society in collaboration with the thoracic society of Australia and New Zealand and the Irish thoracic society. Thorax. 2008;63 Suppl 5:v1-v58.

38. Fechner RE, Greenberg SD, Wilson RK, et al. Evaluation of transbronchial biopsy of the lung. Am J Clin Pathol. 1977;68:17-20.

39. Wall CP, Gaensler EA, Carrington CB, et al. Comparison of transbronchial and open biopsies in chronic infiltrative lung diseases. Am Rev Respir Dis. 1981;123:280-5.

40. Margaritopoulos GA, Wells AU. The role of transbronchial biopsy in the diagnosis of diffuse parenchymal lung diseases: Con. Rev Port Pneumol. 2012;18:61-3. 
41. Casoni GL, Gurioli C, Chhajed PN, et al. The value of transbronchial lung biopsy using jumbo forceps via rigid bronchoscope in diffuse lung disease. Monaldi Arch Chest Dis. 2008;69:59-64.

42. Utz JP, Ryu JH, Douglas WW, et al. High short-term mortality following lung biopsy for usual interstitial pneumonia. Eur Respir J. 2001;17:175-9.

43. Flaherty KR, Travis WD, Colby TV, et al. Histologic variability in usual and nonspecific interstitial pneumonias. Am J Respir Crit Care Med. 2001;164:1722-7.

44. Monaghan H, Wells AU, Colby TV, et al. Prognostic implications of histologic patterns in multiple surgical lung biopsies from patients with idiopathic interstitial pneumonia. Chest. 2004; 125:522-6.
45. Nicholson AG, Addis BJ, Bharucha $\mathrm{H}$, et al. Interobserver variation between pathologists in diffuse parenchymal lung disease. Thorax. 2004;59:500-5.

46. •• Myers JL. Hypersensitivity pneumonia: the role of lung biopsy in diagnosis and management. Mod Pathol. 2012;25:58-67. A detailed overview on histologic findings in HP. The range of patterns has caused diagnostic confusion, especially if biopsy findings are regarded as a diagnostic gold standard. A change in diagnosis from IPF to HP, based on clinical and radiologic features, is a major cause of changes in the histologic diagnosis with multidisciplinary evaluation.

47. Latsi PI, du Bois RM, Nicholson AG, et al. Fibrotic idiopathic interstitial pneumonia: the prognostic value of longitudinal functional trends. Am J Respir Crit Care Med. 2003;168:531-7. 